\title{
A prospective comparative study of hip resurfacing arthroplasty and large-diameter head metal-on-metal total hip arthroplasty in younger patients-a minimum of five year follow-up
}

\author{
Ran Tao $^{1} \cdot$ Fan Liu $^{1} \cdot$ Ya-ke Liu $^{1}$ (D) $\cdot$ Yue Lu ${ }^{1} \cdot$ Hua Xu ${ }^{1} \cdot$ Yi Cao $^{1} \cdot$ Zhen-yu Zhou $^{1} \cdot$ Wei Wang $^{1}$
}

Received: 29 December 2017 / Accepted: 29 January 2018 / Published online: 18 February 2018

(C) The Author(s) 2018. This article is an open access publication

\begin{abstract}
Introduction Both hip resurfacing arthroplasty (HRA) and large-diameter head metal-on-metal total hip arthroplasty (LDH MoM THA) are generally used for young and active patients. A number of comparative studies of HRA and total hip arthroplasty have been published in the literature. However, studies that have compared HRA with LDH MoM THA are rare. The purpose of this study is to compare the mid-term results of HRA with those of LDH MoM THA in young patients.

Patients and methods Between 2007 and 2011, 68 patients were enrolled in the study and randomized into two groups: HRA group (28 hips) and LDH MoM THA group (40 hips). Peri-operative data including blood loss, surgery duration, size of the implant, and post-operative complications were recorded. All patients were assessed clinically and radiologically at six weeks; one, three and five years; and at the time of final review. Functional outcome were assessed using Harris hip (HHS), University of California Los Angeles (UCLA) and Oxford hip (OHS) scores. The mean follow-up for all patients was 7.4 years (5 to 9).

Results Patient groups matched similarly in age, percent female, body mass index, preoperative HHS, and follow-up time. No differences were observed between the two groups in blood loss or in head size or acetabular inclination angle. HRA group had significantly longer surgery duration but less blood loss. The two groups had comparable HHS, UCLA, and OHS at the latest follow-up. Major complications, such as fracture, dislocation, infection, and adverse reactions to the metal debris (ARMD) were not found in the two groups. Only one case in LDH MoM THA group underwent revision surgery due to unexplained pain. Conclusion Comparison of HRA and LDH MoM THA shows similar mid-term clinical results. HRA may be preferable due to the well-preserved bone stock and restoration of the native anatomy. LDH MoM THA may be used with caution due to the excessive metal ion release.
\end{abstract}

Keywords Hip $\cdot$ Arthroplasty $\cdot$ Large diameter head $\cdot$ Resurfacing

\section{Introduction}

Total hip arthroplasty (THR) is the treatment of choice for many patients with end-stage hip diseases. However, conventional THR using metal-on-polyethylene bearings has had a common problem of high incidence of revision due to wear debris $[2,14]$. Metal-on-metal bearings were subsequently introduced to reduce wear and osteolysis and were thought to be suitable for young and active patients.

Ya-ke Liu

18306298834@163.com

1 Department of Orthopaedics, Affiliated Hospital to Nantong University, 20 Xisi Road, Nantong, Jiangsu Province, China
Hip resurfacing arthroplasty (HRA) and large-diameter head metal-on-metal total hip arthroplasty (LDH MoM THA) are the mainly used implants with these bearings and have been popular during the last decade. Recently, a number of published literatures have reported adverse reaction to metal debris (ARMD) which may, at least in part, result in early failure of large-diameter MoM implants [11, $12,18]$. Some large-diameter MoM devices have been recalled, and the use of these devices has dramatically decreased. Yet, the fact is surgeons differ in their use of HRA and LDH MoM THA. Favorable short- to long-term clinical results make many surgeons consider HRA as an effective surgical intervention in a selected population of young patients [3, 8, 13, 16, 23-25]. On the other hand, the use of LDH MoM THA is discontinued because of high incidence of early failure due to the taper wear [10]. 
The purpose of this study is to compare the mid-term results of HRA with those of $\mathrm{LDH}$ in young patients. As we know, few authors reported prospective study concerning comparison of these large-diameter MoM implants.

\section{Materials and methods}

Between 2007 and 2011, 100 young patients ( $\leq 65$ years) who were considered eligible for both HRA and LDH MoM THA were randomized into two groups. Each group consisted of 50 patients. Randomization was undertaken using sealed envelopes which were opened on the day of admission. Selection criteria were avascular necrosis involving less than one-half of the femoral head on a plain radiograph, primary osteoarthritis, developmental dysplasia of hip (DDH) with relatively normal hip morphology, and rheumatoid arthritis. We excluded patients who expressed that they had intention of becoming pregnant, who had renal function insufficiency or bilateral hip replaced. We excluded patients who had bilateral hip replaced. These patients were informed about HRA and LDH MoM THA and willing to participate in the study. However, only 75 patients signed an informed consent on the day before operation. Seventeen patients in HRA group and eight patients in LDH MoM THA group refused to undergo metal-onmetal arthroplasty and chose ceramic-on-polyethylene or ceramic-on-ceramic bearings. Five patients in HRA group and two patients in LDH MoM THA group were lost to a minimum of five year follow-up, respectively. Finally, 68 patients made up the present study. Our institutional review board approved the study.

The implant used was Conserve Plus (Wright Medical Technology, Arlington, TN, USA) in HRA group and Cocr (Wright Medical Technology, Arlington, TN, USA) in LDH MoM THA group, respectively. A uniform posterolateral approach was used in all cases. Good exposure is essential for satisfactory component alignment. The intention was to achieve $45^{\circ}$ of acetabular component inclination, a $5^{\circ}$ to $10^{\circ}$ more anteversion aligned with the native acetabulum, and slight femoral valgus. When HRA was performed, care was taken to avoid notching and excessive load of the femoral neck. All operations were performed by a single surgeon (Fan L). Peri-operative data including blood loss, surgery duration, size of the implant, and postoperative complications were recorded. All patients were assessed clinically and radiologically at six weeks; one, three and five years, and at the time of final review. Computed tomography (CT) scanning was performed at five years and at the time of final review. Functional outcome were assessed using Harris hip (HHS), University of California Los Angeles (UCLA) and Oxford hip (OHS) scores. The mean follow-up for all patients was 7.4 years (5 to 10 ). All statistical analysis was performed using SPSS 17.0. A $p$ value $<0.05$ was used as a threshold for significance. The patient demographics are listed in Table 1.
Table 1 Demographic information

\begin{tabular}{lll}
\hline & $\begin{array}{l}\text { HRA group (28hips) } \\
P \text { value }\end{array}$ & LDH MoM THA group (40 hips) \\
\hline $\begin{array}{l}\text { Gender } 0.955 \\
\text { Male }\end{array}$ & $19(67.9 \%)$ & $28(70.0 \%)$ \\
Female & $9(32.1 \%)$ & $12(30.0 \%)$ \\
Age 0.156 & $43(21$ to 65$)$ & $47(24$ to 65$)$ \\
Mean (range) in years & $21.5(17.8$ to 25.7$)$ & $21.8(17.2$ to 26.2$)$ \\
Body mass index 0.725 & $18(64.3 \%)$ & $32(80.0 \%)$ \\
Mean (range) & $7(25.0 \%)$ & $5(12.5 \%)$ \\
Diagnosis 0.328 & $2(7.1 \%)$ & $3(7.5 \%)$ \\
Avascular necrosis & $1(3.6 \%)$ & \\
Primary osteoarthritis & & $49.2(42$ to 59$)$ \\
Developmental dysplasia & $50.5(41$ to 62$)$ & $7.3(5.3$ to 9.6$)$ \\
Rheumatoid arthritis & $7.7(5.0$ to 10.0$)$ & \\
Harris hip score before surgery 0.257 & &
\end{tabular}


Fig. 1. The two groups had comparable HHS, UCLA, and OHS at the latest follow-up

\section{Results}

Patient groups matched similarly in age, percent female, body mass index, preoperative HHS, and follow-up time. No differences were observed between the two groups in blood loss or in head size or acetabular inclination angle. HRA group had significantly longer surgery duration but less blood loss. The two groups had comparable HHS, UCLA, and OHS at the latest follow-up (Fig. 1). Comparison of clinical outcomes between the two groups was seen in Table 2. Good exposure is essential for satisfactory component alignment. The intention was to achieve $45^{\circ}$ of acetabular component inclination, a $5^{\circ}$ to $10^{\circ}$ more anteversion aligned with the native acetabulum, and slight femoral valgus. When HRA was performed, care was taken to avoid notching and excessive load of the femoral neck. Major complications, such as fracture, dislocation, infection, and ARMD were not found in the two groups. Only one case in LDH MoM THA group underwent revision surgery five years after

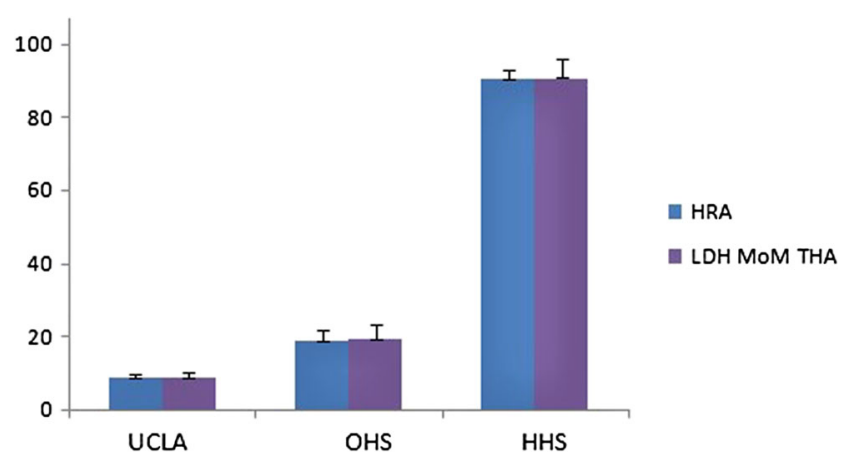

primary surgery due to unexplained pain. The 65-yearold gentleman complained of persistent hip pain five years after the index procedure, without any sign of ARMD or another major complication. He underwent revision surgery in another hospital and was unwilling to keep in touch with us because of the poor outcome (Figs. 2 and 3 ).

\section{Discussion}

We find comparable functional results between the groups in a minimum of five year follow-up, except for the revision case in LDH MoM THA group. Garbuz et al. [6] also compared the two metal-on-metal bearings and reported similar short-term results. The authors further warned against the use of LDH MoM THA due to the excessive metal ion release. ARMD is the major complication following large-diameter head metalon-metal hip arthroplasty. The clinical findings of ARMD may include periarticular fluid collections, soft tissue masses,
Table 2 Comparison of clinical outcomes in the two groups

\begin{tabular}{lll}
\hline $\begin{array}{l}\text { (28 hips) } \\
P \text { value }\end{array}$ & HRA group & LDH MoM THA group (40 hips) \\
\hline Blood loss (ml) 0.002 & $353 \pm 79$ & $429 \pm 109$ \\
Surgery duration (min) 0.00 & $98 \pm 12$ & $79 \pm 9$ \\
Head size (mm) 0.531 & $46.4 \pm 2.5$ & $46.4 \pm 2.5$ \\
$42 \mathrm{~mm}$ & $1(3.6 \%)$ & $2(5.0 \%)$ \\
$44 \mathrm{~mm}$ & $9(32.1 \%)$ & $11(27.5 \%)$ \\
$46 \mathrm{~mm}$ & $7(25.0 \%)$ & $12(30.0 \%)$ \\
$48 \mathrm{~mm}$ & $6(21.4 \%)$ & $11(27.5 \%)$ \\
$50 \mathrm{~mm}$ & $4(14.3 \%)$ & $1(2.5 \%)$ \\
$52 \mathrm{~mm}$ & $1(3.6 \%)$ & $3(7.5 \%)$ \\
Acetabular inclination angle (AIA) 0.274 & $50 \pm 6$ & $48 \pm 5$ \\
Functional outcome at latest follow-up & & \\
HHS 0.708 & $90.4 \pm 2.4$ & $90.8 \pm 5.1$ \\
UCLA 0.479 & $8.5 \pm 1.0$ & $8.7 \pm 1.3$ \\
OHS 0.714 & $18.7 \pm 3.0$ & $19.1 \pm 4.4$ \\
\hline
\end{tabular}

$H H S$, Harris hip score; UCLA, University of California, Los Angeles; OHS, Oxford Hip score 

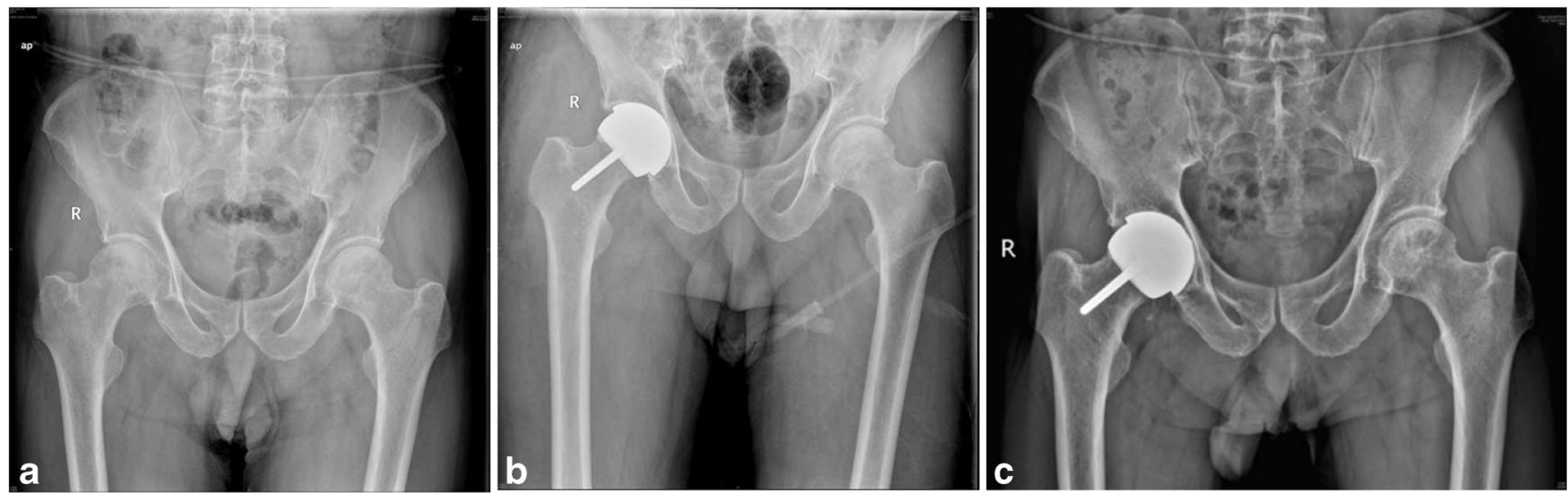

Fig. 2 A 65-year-old gentleman with bilateral femoral head necrosis underwent HRA on the right side in 2008. Pelvic radiographs: a preoperation, b 5 days post operation, $\mathbf{c} 9$ years post operation. Notice that the left femoral head did not collapse over time

and gluteal muscle necrosis [18]. Some authors suspected that clicking and a sensation of subluxation was correlated to the later failure of the devices. In the present study, however, both patients experienced such symptoms obtained good results with negative CT findings. In the present study, ARMD was not observed in the two groups. Magnetic resonance imaging (MRI), especially metal artifact reduction sequence magnetic resonance imaging (MARS MRI), and slice encoding for metal artifact correction (SEMAC MRI) is effective in detecting patients with ARMD [24]. However, such techniques are unavailable in our center. This is one of the limitations of this study.

Numerous published literatures $[5,9,16,22,25]$ reported excellent short- to mid-term outcomes in HRA. The significantly longer surgical time in HRA group can be explained by more complexity and higher technique demanding of this procedure. The significantly less blood loss may be attributed to the intact femur medullary cavity. Sershon et al. [24] summarized femoral neck fracture and aseptic loosening as the two most common modes of failure in FDA-approved HRA. We do not have cases of failure mainly due to meticulous protection of femoral neck during surgery and relatively short-term follow-up. On the other hand, the clinical outcomes following LDH MoM THA are discouraging. Many authors reported early failures of LDH MoM THA [11, 12, 19]. Extensive corrosion on the taper and trunnion was observed in LDH MoM THA revisions. This probably contributed to excessive metal debris releasing and ARMD [17]. In the present study, however, all hips functioned well except the revision one at five year follow-up. Longer follow-up is needed for these cases. Both HRA and LDH MoM THA are large-diameter head metal-on-metal arthroplasty. The latter one appears to release more metal ions [10]. We did not measure serum levels of cobalt and chromium ions. This is another limitation of this study.

The main subjects of many comparative studies between HRA and total hip arthroplasty are patients with osteoarthritis. In this study cohort, the majority are patients with avascular necrosis of femoral head (64.3\% in HRA group and $80 \%$ in LDH MoM THA group, respectively). Some authors do not
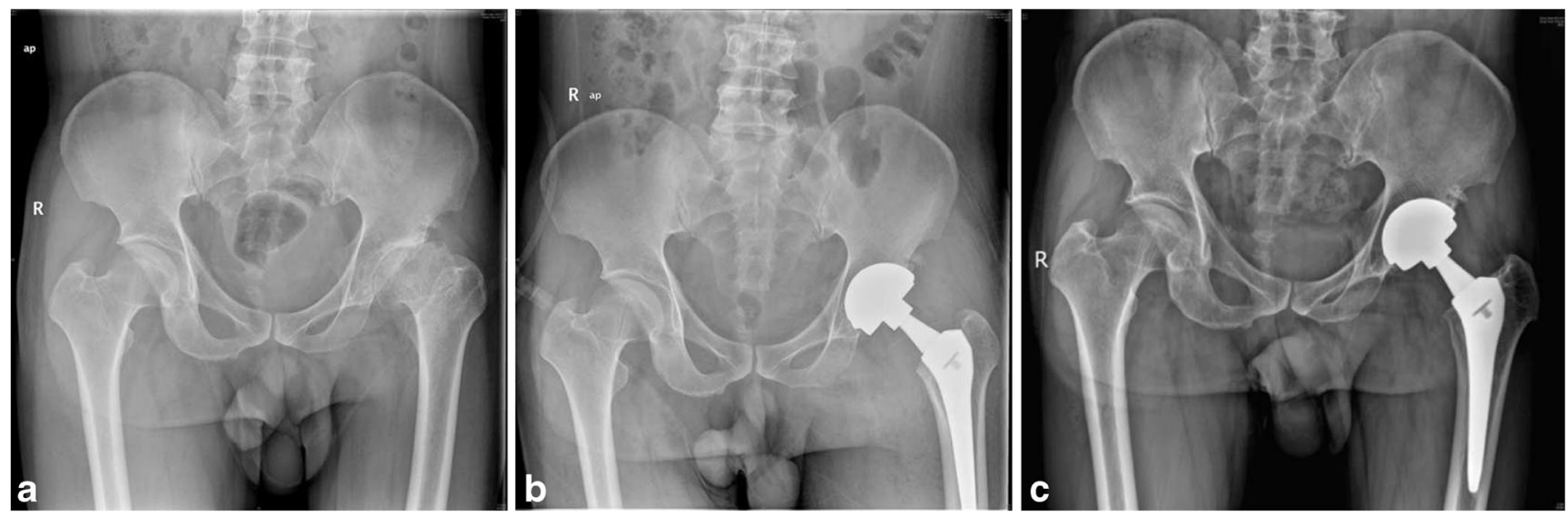

Fig. 3 A 46-year-old farmer sustained left hip osteoarthritis secondary to DDH. LDH MoM THA was performed in 2008. Pelvic radiographs: a preoperation, $\mathbf{b} 5$ days post operation, $\mathbf{c} 8.5$ years post operation 
think avascular necrosis of femoral head is indicated for HRA [7]. Our study demonstrated good mid-term results. We believe it a good indication for HRA and this is in accordance with other authors' opinion $[1,15,21,23,24]$. DDH may be an independent risk factor for failure following HRA, since the vertical, anteverted nature of the dysplastic hip results in significant cup uncoverage at the anterosuperior edge when the cup is placed in the recommended $40^{\circ}$ of abduction. In the present study, the only two dysplastic hips we selected had relatively normal hip morphology. The cup coverage was acceptable and the hips functioned well.

The strengths of this study are twofold. First, it is a randomized prospective study. Haddad et al. [8] emphasized on the difficulties in randomized comparative study between HRA and THA. In their study, only 24 patients were randomized, and we have 68 randomized patients. Second, we followed patients at a minimum of five years, while many comparative studies between HRA and THA are short-term studies $[4,6,20]$.

In conclusion, comparison of HRA and LDH MoM THA shows similar mid-term clinical results. HRA may be preferable due to the well-preserved bone stock and restoration of the native anatomy. However, patient selection, surgical technique, as well as implant design should be taken into consideration. LDH MoM THA may be used carefully due to the excessive metal ion releasing.

\section{Compliance with ethical standards}

Conflict of interest The authors declare that they have no conflict of interest.

Open Access This article is distributed under the terms of the Creative Commons Attribution 4.0 International License (http:// creativecommons.org/licenses/by/4.0/), which permits unrestricted use, distribution, and reproduction in any medium, provided you give appropriate credit to the original author(s) and the source, provide a link to the Creative Commons license, and indicate if changes were made.

\section{References}

1. Amstutz HC, Le Duff MJ (2016) Hip resurfacing for osteonecrosis: two- to 18-year results of the conserve plus design and technique. Bone Joint J 98-B:901-909

2. Barrack RL, Folgueras A, Munn B et al (1997) Pelvic lysis and polyethylene wear at 5-8 years in a cementless total hip. Clin Orthop Relat Res 335:211-217

3. Brooks PJ (2016) Hip resurfacing: a large, US single-surgeon series. Bone Joint J 98-B(1 Suppl A):10-13

4. Costa ML, Achten J, Parsons NR et al (2012) Total hip arthroplasty versus resurfacing arthroplasty in the treatment of patients with arthritis of the hip joint: single centre, parallel group, assessor blinded, randomised controlled trial. BMJ 344:2147

5. Daniel J, Pradhan C, Ziaee H et al (2014) Results of Birmingham hip resurfacing at 12 to 15 years: a single-surgeon series. Bone Joint J 96-b(10):1298-1306
6. Garbuz DS, Tanzer M, Greidanus NV et al (2010) The John Charnley Award: metal-on-metal hip resurfacing versus largediameter head metal-on-metal total hip arthroplasty: a randomized clinical trial. Clin Orthop Relat Res 468:318-325

7. Gross TP, Liu F (2012) Comparative study between patients with osteonecrosis and osteoarthritis after hip resurfacing arthroplasty. Acta Orthop Belg 78(6):735-744

8. Haddad FS, Konan S, Tahmassebi J (2015) A prospective comparative study of cementless total hip arthroplasty and hip resurfacing in patients under the age of 55 years: a ten-year follow-up. J Bone Joint Surg (Br) 97-B:617-622

9. Holland JP, Langton DJ, Hashmi M (2012) Ten-year clinical, radiological and metal ion analysis of the Birmingham Hip Resurfacing: from a single, non-designer surgeon. J Bone Joint Surg (Br) 94(4:471-476

10. Lainiala OS, Moilanen TPS, Hart AJ et al (2016) Higher blood cobalt and chromium levels in patients with unilateral metal-onmetal total hip arthroplasties compared to hip resurfacings. J Arthroplast 31:1261-1266

11. Langton DJ, Jameson SS, Joyce TJ et al (2010) Early failure of metal-onmetal bearings in hip resurfacing and larger-diameter total hip replacement: a consequence of excess wear. J Bone Joint Surg (Br) 92-B:38

12. Langton DJ, Joyce TJ, Jameson SS et al (2011) Adverse reaction to metal debris following hip resurfacing: the influence of component type, orientation and volumetric wear. J Bone Joint Surg (Br) 93-B:164

13. Lons A et al (2015) Excellent short-term results of hip resurfacing in a selected population of young patients. Orthop Traumatol Surg Res. https://doi.org/10.1016/j.otsr

14. Mäkelä KT, Eskelinen A, Pulkkinen P et al (2011) Results of 3,668 primary total hip replacements for primary osteoarthritis in patients under the age of 55 years. Acta Orthop 82:521

15. Matharu GS, Pandit HG, Murray DW et al (2015) The future role of metal-on-metal hip resurfacing. Int Orthop (SICOT) 39:2031-2036

16. Mehra A, Berryman F, Matharu GS et al (2015) Birmingham hip resurfacing: a single surgeon series reported at a minimum of 10 years follow-up. J Arthroplasty. https://doi.org/10.1016/j.arth.2015.01.042

17. Meyer H, Mueller T, Goldau G et al (2012) Corrosion at the cone/ taper interface leads to failure of large-diameter metal-on-metal total hip arthroplasties. Clin Orthop 11:3101-3108

18. Mokka J, Junnila M, Seppänen M et al (2013) Adverse reaction to metal debris after ReCap-M2A-magnum large-diameter-head metal-on-metal total hip arthroplasty. Acta Orthop 84(6):549-554

19. Mokka J, Mäkelä KT, Virolainen P et al (2013) Cementless total hip arthroplasty with large diameter metal-on-metal heads - short-term survivorship of 8,059 hips from the Finnish Arthroplasty Register. Scand J Surg 102(2):117-123

20. Mont MA, Marker DR, Smith JM, Ulrich SD, McGrath MS (2009) Resurfacing is comparable to total hip arthroplasty at short-term follow-up. Clin Orthop Relat Res 467:66-71

21. Nakasone S, Takao M, Sakai T et al (2013) Does the extent of osteonecrosis affect the survival of hip resurfacing? Clin Orthop Relat Res 471(6):1926-1934

22. Nam D, Nunley RM, Ruh EL et al (2015) Short-term results of Birmingham hip resurfacing in the United States. Orthopedics 38(8):e715-e721

23. Pyda M, Koczy B, Widuchowski W et al (2015) Hip resurfacing arthroplasty in treatment of avascular necrosis of the femoral head. Med Sci Monit 21:304-309

24. Sershon R, Balkissoon R, Valle CJD et al (2016) Current indications for hip resurfacing arthroplasty in 2016. Curr Rev Musculoskelet Med 9:84-92

25. Su EP, Housman LR, Masonis JL et al (2014) Five year results of the first US FDA-approved hip resurfacing device. J Arthroplast 29: $1571-1575$ 